\title{
GROWTH on S190510g: DECam Observation Planning and Follow-up of a Distant Binary Neutron Star Merger Candidate
}

\author{
Igor Andreoni ${ }^{1,19}$ (i) , Daniel A. Goldstein ${ }^{1,19,20}$ (10), Shreya Anand ${ }^{1}$, Michael W. Coughlin ${ }^{1}$ (i), Leo P. Singer ${ }^{2,3}$ (D),

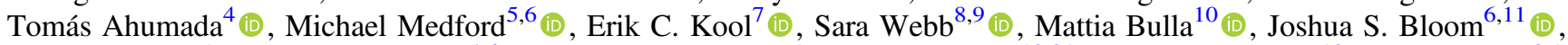

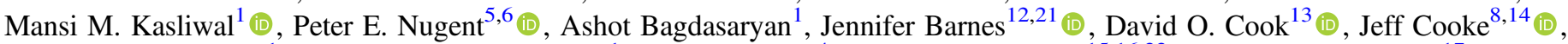 \\ Dmitry A. Duev ${ }^{1}$ (1D) U. Christoffer Fremling ${ }^{1}$, Pradip Gatkine ${ }^{4}$ (D), V. Zach Golkhou ${ }^{15,16,22}$ (D) , Albert K. H. Kong ${ }^{17}$ (1), \\ Ashish Mahabal ${ }^{1,18}$ (1) , Jorge Martínez-Palomera ${ }^{6}$, Duo Tao ${ }^{1}$, and Keming Zhang (张可名) ${ }^{6}$ (1) \\ ${ }^{1}$ Division of Physics, Mathematics, and Astronomy, California Institute of Technology, Pasadena, CA 91125, USA; andreoni@ caltech.edu \\ ${ }_{2}^{2}$ Astrophysics Science Division, NASA Goddard Space Flight Center, MC 661, Greenbelt, MD 20771, USA \\ ${ }^{3}$ Joint Space-Science Institute, University of Maryland, College Park, MD 20742, USA \\ ${ }^{4}$ Department of Astronomy, University of Maryland, College Park, MD 20742, USA \\ ${ }^{5}$ Computational Science Department, Lawrence Berkeley National Laboratory, 1 Cyclotron Road, MS 50B-4206, Berkeley, CA 94720, USA \\ ${ }^{6}$ Department of Astronomy, University of California, Berkeley, CA 94720-3411, USA \\ ${ }^{7}$ The Oskar Klein Centre \& Department of Astronomy, Stockholm University, AlbaNova, SE-106 91 Stockholm, Sweden \\ ${ }^{8}$ Centre for Astrophysics and Supercomputing, Swinburne University of Technology, Hawthorn, VIC, 3122, Australia \\ ${ }^{9}$ ARC Centre of Excellence for Gravitational Wave Discovery (OzGrav), Australia \\ ${ }^{10}$ The Oskar Klein Centre, Department of Physics, Stockholm University, AlbaNova, SE-106 91 Stockholm, Sweden \\ ${ }^{11}$ Lawrence Berkeley National Laboratory, 1 Cyclotron Road, MS 50B-4206, Berkeley, CA 94720, USA \\ ${ }^{12}$ Columbia Astrophysics Laboratory, Columbia University, New York, NY 10032, USA \\ ${ }^{13}$ IPAC, California Institute of Technology, 1200 E. California Blvd, Pasadena, CA 91125, USA \\ ${ }^{14}$ Australian Research Council Centre of Excellence for Gravitational Wave Discovery (OzGrav), Hawthorn, VIC, 3122, Australia \\ ${ }^{15}$ DIRAC Institute, Department of Astronomy, University of Washington, 391015 th Avenue NE, Seattle, WA 98195, USA \\ ${ }^{17}$ The eScience Institute, University of Washington, Seattle, WA 98195, USA
Institute of Astronomy, National Tsing Hua University, Hsinchu 30013, Taiwan \\ ${ }^{18}$ Center for Data Driven Discovery, California Institute of Technology, Pasadena, CA 91125, USA \\ Received 2019 May 31; revised 2019 July 15; accepted 2019 July 18; published 2019 August 9
}

\begin{abstract}
The first two months of the third Advanced LIGO and Virgo observing run (2019 April-May) showed that distant gravitational-wave $(\mathrm{GW})$ events can now be readily detected. Three candidate mergers containing neutron stars (NS) were reported in a span of 15 days, all likely located more than $100 \mathrm{Mpc}$ away. However, distant events such as the three new NS mergers are likely to be coarsely localized, which highlights the importance of facilities and scheduling systems that enable deep observations over hundreds to thousands of square degrees to detect the electromagnetic counterparts. On 2019 May 10 02:59:39.292 UT the GW candidate S190510g was discovered and initially classified as a binary neutron star (BNS) merger with $98 \%$ probability. The GW event was localized within an area of $3462 \mathrm{deg}^{2}$, later refined to $1166 \mathrm{deg}^{2}(90 \%)$ at a distance of $227 \pm 92 \mathrm{Mpc}$. We triggered Target-ofOpportunity observations with the Dark Energy Camera (DECam), a wide-field optical imager mounted at the prime focus of the $4 \mathrm{~m}$ Blanco Telescope at Cerro Tololo Inter-American Observatory in Chile. This Letter describes our DECam observations and our real-time analysis results, focusing in particular on the design and implementation of the observing strategy. Within $24 \mathrm{hr}$ of the merger time, we observed $65 \%$ of the total enclosed probability of the final skymap with an observing efficiency of $94 \%$. We identified and publicly announced 13 candidate counterparts. S190510g was reclassified 1.7 days after the merger, after our observations were completed, with a "BNS merger" probability reduced from $98 \%$ to $42 \%$ in favor of a "terrestrial classification.
\end{abstract}

Key words: binaries: general - gravitational waves - methods: observational - stars: neutron - supernovae: general

\section{Introduction}

The joint detection of electromagnetic (EM) and gravitational-wave $(\mathrm{GW})$ signals from the binary neutron star (BNS) merger GW170817 (Abbott et al. 2017b) was a watershed moment for astronomy. The discovery of the GW event triggered an extensive EM follow-up campaign, and the resulting panchromatic data set exacted stringent constraints on fundamental physics (Abbott et al. 2017b), gave new insight into the origin of the heavy elements (e.g., Arcavi et al. 2017; Chornock et al. 2017; Coulter et al. 2017; Cowperthwaite et al.

\footnotetext{
19 These authors contributed equally to this work.

${ }^{20}$ Hubble Fellow.

21 Einstein Fellow.

22 Moore-Sloan, WRF, and DIRAC Fellow.
}

2017; Kasen et al. 2017; Kasliwal et al. 2017; Pian et al. 2017; Smartt et al. 2017), demonstrated a novel technique for measuring cosmological parameters (Abbott et al. 2017a), and marked the beginning of the "GW multi-messenger era" (Abbott et al. 2017c).

The Swope Supernova Survey first reported the optical counterpart to GW170817 in NGC 4993 from a galaxy-targeted search (Coulter et al. 2017) with independent confirmation by several other teams shortly after (Arcavi et al. 2017; Lipunov et al. 2017; Soares-Santos et al. 2017; Tanvir et al. 2017; Valenti et al. 2017). Galaxy-targeted searches pre-select galaxies that could harbor counterparts based on criteria such as sky location, distance, star formation rate, or stellar mass, then search those galaxies for transients. As galaxy-targeted searches do not require observations of large swaths of sky, 
they can be carried out on telescopes with small fields of view (see for example Golkhou et al. 2018). The galaxy-targeted approach worked particularly well in case of GW170817 because NGC 4993 is relatively nearby $(D=41.0 \pm 3.1 \mathrm{Mpc}$; Hjorth et al. 2017, but see also Im et al. 2017; Levan et al. 2017; Pan et al. 2017; Cantiello et al. 2018) and the optical counterpart to GW170817 was bright enough $\left(M_{V} \sim-16\right)$ to be detected with $1 \mathrm{~m}$ class telescopes.

However, GW170817 was far better localized, and much closer to Earth, than any of the three neutron star (NS)-bearing compact binary mergers that have been detected in GWs since (The LIGO Scientific Collaboration \& the Virgo Collaboration 2019a, 2019b, 2019c). The newest events have had typical distances of a few hundred megaparsecs, and typical localizations of about $10^{3} \mathrm{deg}^{2}$, orders of magnitude larger than those of GW170817. Detecting the "kilonovae" (optical and infrared transients with evolution timescales of hours to days) associated with BNS mergers at relatively large distances using a galaxy-targeted approach is challenging, as the signal is expected to be dim and galaxy catalogs are incomplete (Cook et al. 2019). For these events, telescopes with large apertures and wide fields of view are required to systematically search for counterparts over large areas of sky. An instrument well suited to this task in the Southern Hemisphere is the Dark Energy Camera (DECam; Flaugher et al. 2015), a $\sim 3 \mathrm{deg}^{2}$ wide-field imager mounted at the prime focus of the $4 \mathrm{~m}$ Blanco telescope at the Cerro Tololo InterAmerican Observatory (CTIO).

A key challenge of the wide-field approach to GW follow-up is determining the optimal sequence of observations to maximize counterpart discovery potential over wide areas of sky. Several different variables, including reference coverage, filter choice, observability, Galactic extinction, event localization, and exposure times must all be taken into account. DECam has been extensively used in the past to follow up GW events (Annis et al. 2016; Cowperthwaite et al. 2016; SoaresSantos et al. 2016; Doctor et al. 2019), with the detection of the counterpart to GW170817 being a particular success (Cowperthwaite et al. 2017; Soares-Santos et al. 2017). The strategy adopted during those follow-up campaigns (described in Soares-Santos et al. 2016) relied on an all-sky mapping of observational parameters from models of sky brightness, atmospheric transmission, interstellar dust extinction, expected seeing, and confusion-limit probability to estimate the probability that putative GW counterparts would be detected by DECam assuming theoretical predictions for the peak luminosity. This information was then combined with the GW skymap to determine the patches of sky to be observed.

In this Letter, we describe an automated approach we have developed to solving the wide-field tiling problem, presenting a real-world application to DECam follow-up observations of the GW event S190510g, carried out as part of the DECamGROWTH component of the Global Relay of Observatories Watching Transients Happen (GROWTH) collaboration. In Section 2, we describe the GW event and its properties. We describe our tiling algorithm, observations, and data analysis methods in Section 3. In Section 4, we present 13 high-priority transients discovered during the real-time analysis that were promptly reported to the community via the Gamma-ray Coordinate Network (GCN). In addition, we present 10 lowerpriority transient candidates for completeness. In Section 5 we discuss both the performance of our scheduling methods and the outcome of our searches.

\section{The Gravitational-wave Event S190510g}

On 2019 May 10 02:59:39.292 UT, the compact binary merger candidate S190510g was discovered with the Advanced LIGO and Virgo detectors in triple coincidence (The LIGO Scientific Collaboration \& the Virgo Collaboration 2019c) using the GstLAL analysis pipeline (Messick et al. 2017). The event was reported to have a false alarm rate of about 1 in 37 $\mathrm{yr}$, and was initially classified as a BNS merger with $98 \%$ probability. The first localization sky map obtained with the BAYESTAR software (Singer \& Price 2016), released on 2019 May 10 04:03:43 UT, constrained the 90\% localization probability to a sky area of $3462 \mathrm{deg}^{2}$. The luminosity distance was reported to be $269 \pm 108 \mathrm{Mpc}$. Based on S190510g's initially high probability of being a BNS merger, we triggered DECam follow-up to search for an optical counterpart. The observations were taken over two consecutive nights, and are described in detail in Section 3.3. The initial and refined skymaps are shown in Figure 1, along with our coverage of the event with DECam (Section 3.3).

A refined skymap from the LALInference localization pipeline (Veitch et al. 2015) was made available on 2019 May 10 10:06:59 UT, between our first and second nights of observations, reducing the $90 \%$ probability region to a sky area of $1166 \mathrm{deg}^{2}$, with $50 \%$ of the integrated probability enclosed in a region only $31 \mathrm{deg}^{2}$ wide and observable with DECam (The LIGO Scientific Collaboration \& the Virgo Collaboration 2019e). The modified luminosity distance was $227 \pm 92 \mathrm{Mpc}$.

On 2019 May 11 20:19:22 UT, after our second and final night of observations had concluded, the LIGO Scientific Collaboration and the Virgo Collaboration (LVC) announced an update on the significance of the S190510g event (The LIGO Scientific Collaboration \& the Virgo Collaboration 2019d). The resulting re-estimate of the background model yielded a false alarm rate of 1 in $3.6 \mathrm{yr}$, significantly higher than the rate of 1 in $37 \mathrm{yr}$ initially reported in The LIGO Scientific Collaboration \& the Virgo Collaboration (2019c). The LVC reclassified S190510g to have a $58 \%$ probability of being terrestrial and a $42 \%$ of being a BNS, increasing the odds that this GW event was not astrophysical.

\section{Tiling Strategy and Observations}

Tiling, time-allocation, and telescope scheduling were handled through gwemopt ${ }^{23}$ (Coughlin et al. 2018), a code designed for scheduling observations of GW skymaps with wide-field imagers. The code divides the GW healpix skymap into "tiles" of the size of the field of view of DECam, approximated to circles with 0.9 radii. The code then determines the available segments for observation of each tile throughout the night, and applies a scheduling algorithm to the tiles to generate the order for observation. The user can select among several scheduling algorithms while planning observations. For each designed plan, the code calculates and displays summary statistics featuring the expected probability coverage, area tiled, and total time spent observing for each event. We

\footnotetext{
23 https://github.com/mcoughlin/gwemopt
} 

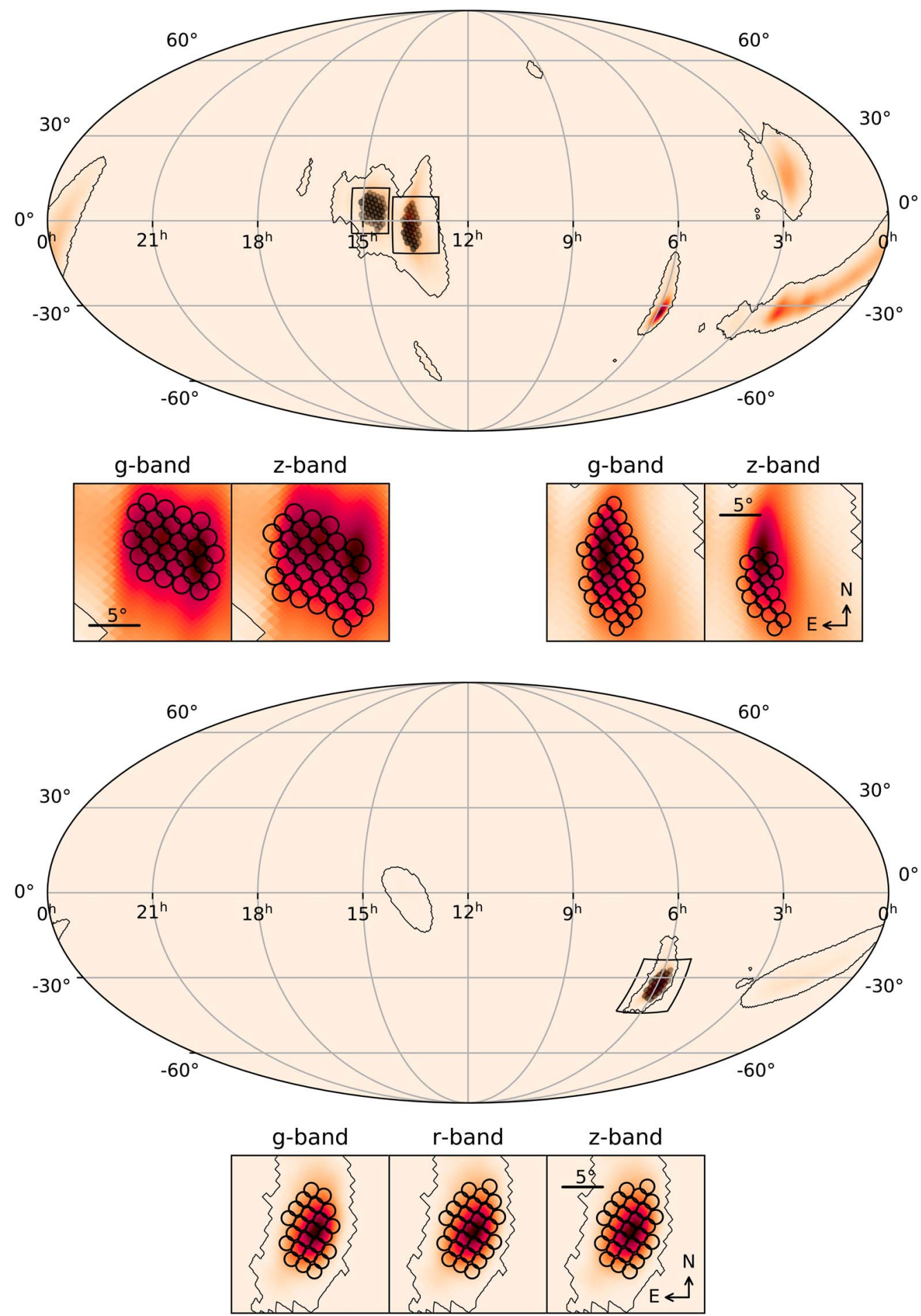

Figure 1. Preliminary BAYESTAR (top, The LIGO Scientific Collaboration \& the Virgo Collaboration 2019c) and refined LALInference (bottom, The LIGO Scientific Collaboration \& the Virgo Collaboration 2019e) localization probability skymaps of the GW event S190510g. Circles show the DECam coverage, approximating the DECam FoV using a radius of $0.9 \mathrm{deg}^{2}$. We based our follow-up on the BAYESTAR map on the first night and the LALInference map on the second night, when it became available. The high-probability patch at R.A. $\sim 6 \mathrm{hr}$, where most of the probability of the LALInference skymap lies, had already set in Chile when the GW event occurred on the first night.

use this information to evaluate the performance of different plans in covering the accessible sky-error region.

\subsection{Scheduling Algorithms}

The "greedy" algorithm is the default algorithm used for scheduling observations with gwemopt for most of the telescopes used by GROWTH including DECam and the Zwicky Transient Facility (Bellm et al. 2019, ZTF). The greedy algorithm selects the highest-probability tile that is available for observation within a given time window while taking into account setting constraints (Rana et al. 2017). While particularly effective for small-mount telescopes, the greedy algorithm can show significant limitations when long slews or slow filter 

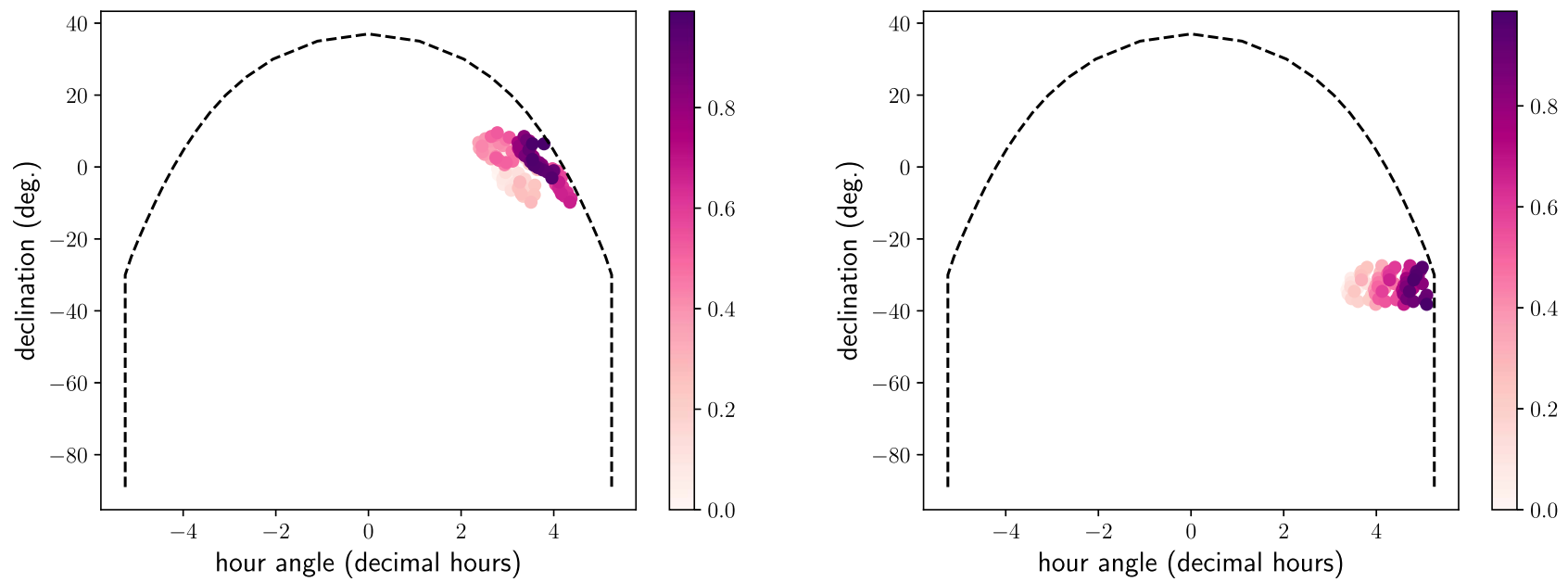

Figure 2. Hour angle as a function of decl. These plots show the performance of the greedy-slew algorithm in scheduling observations on the first and second night within the hour-angle constraint for the Blanco Telescope (CTIO). The color bar, from light to dark, indicates the chronological sequence of exposures taken. Due to an unanticipated system failure and a choice of overhead times too tight to accommodate such unexpected delays, our schedule hit the hour-angle limit during the first night of observations. On the second night, with overheads optimally estimated, we could conduct 3 epochs of observations before pushing the hour-angle limit.

changes are expected (Rana et al. 2019). The main constraints that the Blanco telescope engineering imposes on the scheduler are a slewing rate of $\sim 1 \mathrm{deg} \mathrm{s}^{-1}$ and a firm limit on the observable hour angle as a function of the decl. (see Figure 2).

As a result, we developed the "greedy-slew" algorithm, optimized for Blanco/DECam observations. The greedy-slew algorithm is based on the greedy algorithm, but in addition it takes the Blanco hour-angle constraints into account and it penalizes large slews. Instead of selecting a tile purely based on the probability criteria, the greedy-slew algorithm weights the probabilities based on the ratio between the readout and slew time, so that higher probability tiles with smaller slews are assigned a higher priority. Further optimization of the greedyslew algorithm is in progress.

\subsection{Baseline Observing Strategy}

The baseline strategy for BNS merger follow-up for our program was designed for events placed at $120 \pm 30 \mathrm{Mpc}$ distance, the nominal angle-averaged horizon, or BNS range, of the GW detectors network during O3 (Abbott et al. 2018). The baseline strategy consists of $g-z-g$ blocks of observations on the first night after the $\mathrm{GW}$ trigger, followed by $g-z$ blocks on the second night after the merger. Planned exposure times are $15 \mathrm{~s}$ in $g$ and $25 \mathrm{~s}$ in $z$ and sky regions where template images are available and are preferred to those without pre-imaging. The exposure times were chosen based on GW170817-like kilonova models (Barnes et al. 2016). This baseline strategy was designed to rapidly identify optical counterparts via the measurement of the intra-night and inter-night color evolution of kilonovae that are expected to evolve at a much faster rate than supernovae (see, for example, Kilpatrick et al. 2017; Shappee et al. 2017).

\subsection{Observations}

Our follow-up observations of S190510g were performed under the National Optical Astronomy Observatory (NOAO) proposal ID 2019A-0205 (PIs Goldstein and Andreoni). We announced the start of the observations and the availability of public DECam data via GCN (Andreoni et al. 2019b).
We planned DECam observations using the GROWTH target-of-opportunity (ToO) Marshal ${ }^{24}$ (Coughlin et al. 2019; Kasliwal et al. 2019), an open source web platform developed by the GROWTH team for the follow-up of multi-messenger transients including GW events, neutrino events, and short gamma-ray bursts. The GROWTH ToO Marshal ingests multimessenger triggers and allows the user to plan observations for five facilities (DECam, ZTF, Palomar Gattini-IR, the Kitt Peak Electron Multiplying CCD demonstrator, and the GROWTH India Telescope) using programmatic follow-up algorithms. We planned the observations described in this Letter using the greedy-slew algorithm described in Section 3.1.

On the first night (hereafter "night 1") the BAYESTAR skymap indicated that the $90 \%$ and $50 \%$ probability regions were $3462 \mathrm{deg}^{2}$ and $575 \mathrm{deg}^{2}$, respectively. We started acquiring photons on 2019-10 06:00:25, or $3.01 \mathrm{hr}$ after the merger, performing a block of $g$-band observations followed by a block of $z$-band observations, using $30 \mathrm{~s}$ exposures in each band. Assuming an effective field of view of $2.68 \mathrm{deg}^{2}$ that accounts for the chip gaps, we covered $174.20 \mathrm{deg}^{2}$ in $g$ and $144.72 \mathrm{deg}^{2}$ in $z$ bands, covering $15.18 \%$ and $10.23 \%$ integrated probability respectively in each band. We chose against adopting a dithering pattern to cover the chip gaps in favor of a larger sky coverage.

After our night 1 observations were completed, a new LALInference skymap moved the highest-probability region away from the part of the sky observed on night 1 , reducing the covered integrated probability to only $\sim 2 \%$. As Figure 1 shows, the new skymap instead favored a bulge located at $\mathrm{R}$. A. $\sim 6 \mathrm{hr}$, decl. $\sim-35^{\circ}$ (The LIGO Scientific Collaboration $\&$ the Virgo Collaboration 2019e). This high-probability region of sky had already set at CTIO when the merger occurred on night 1 , so we could not use DECam to acquire early followup data.

The refined skymap constrained the highest-probability region (90\% and 50\% integrated probability being included in $1166 \mathrm{deg}^{2}$ and $31 \mathrm{deg}^{2}$, respectively) to be visible for $\sim 1.6 \mathrm{hr}$ at the beginning on the Chilean night on 2019 May 10 UT. The template coverage in the sky area with top $50 \%$ priority was

\footnotetext{
${ }^{24}$ https://github.com/growth-astro/growth-too-marshal
} 
Table 1

Summary of Observations

\begin{tabular}{|c|c|c|c|c|c|c|c|c|c|c|}
\hline & $\begin{array}{c}\text { Time Start } \\
\text { UT }\end{array}$ & $\begin{array}{c}\text { Time End } \\
\text { UT }\end{array}$ & Filter & $n_{\exp }$ & $\begin{array}{l}t_{\exp } \\
(\mathrm{s})\end{array}$ & $\begin{array}{c}\text { Prob } \\
(\%)\end{array}$ & $\begin{array}{c}\text { Area } \\
\left(\operatorname{deg}^{2}\right)\end{array}$ & $\begin{array}{c}t_{\mathrm{tot}} \\
\text { (minute) }\end{array}$ & $\epsilon_{20}$ & $\epsilon_{30}$ \\
\hline night 1 & 2019 May 10 06:00:25 & 2019 May 10 07:10:02 & $g$ & 65 & 30 & 15.18 & 174.20 & 70.0 & 0.78 & 0.93 \\
\hline night 1 & 2019 May 10 07:12:12 & 2019 May 10 08:22:40 & $z$ & 54 & 30 & 10.23 & 144.72 & 70.5 & 0.64 & 0.77 \\
\hline night 2 & 2019 May 10 22:51:57 & 2019 May 10 23:25:50 & $z$ & 56 & 40 & 65.02 & 75.04 & 33.9 & 0.83 & 0.96 \\
\hline night 2 & 2019 May 10 23:27:09 & 2019 May 11 00:02:10 & $r$ & 56 & 40 & 65.02 & 75.04 & 35.0 & 0.80 & 0.93 \\
\hline night 2 & 2019 May 11 00:03:27 & 2019 May 11 00:31:40 & $g$ & 24 & 40 & 62.63 & 64.32 & 28.2 & 0.85 & 0.99 \\
\hline
\end{tabular}

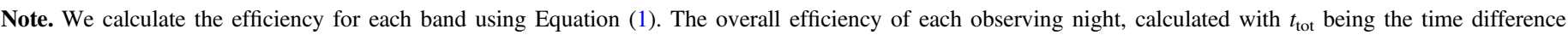
between the last and the first exposure of the ToO observations, amounts to $\epsilon=0.70$ on night 1 and $\epsilon=0.80$ on night 2 .

90\% complete in all filters from Dark Energy Survey (DES) Data Release 1 (DR1) pre-imaging. On the second night (hereafter "night 2") we commenced observations on 2019 May 10 22:51:57 and finished on 2019 May 11 00:31:40, when the field set. We performed blocks of observations in $z-r-g$ bands using $40 \mathrm{~s}$ exposures in each band. We observed $75.04 \mathrm{deg}^{2}$ of effective sky area in $z$ and $r$, covering $65 \%$ of the skymap integrated probability in each band. We then observed $64.32 \mathrm{deg}^{2}$ in the $g$ band, covering $62.3 \%$ of the skymap integrated probability.

Other viable observing options for night 2 (that we did not select) included: (i) observing a larger sky area in two filters with the same exposure time; (ii) increasing the exposure time for at least one filter; (iii) performing a second pass in a certain band of the observed tiles; (iv) using the $i$ band instead of the $g$ band for the third block of observations. We excluded (i) because spending the last 28 minutes of observations imaging a lower-probability region would have yielded only $\sim 2 \%$ additional integrated probability. In order to increase the depth in the $z$ band, the less sensitive among the preferred filters, we considered options (ii) and (iii), either with longer exposures or via image stacking of multiple exposures. We note that two $z$ band epochs acquired on night 2 that were less than $2 \mathrm{hr}$ apart were not expected to return useful information about the transient evolution, unlike $g$-band epoch pairs a few hours after the merger. The last option (iv), involving $i$-band exposures, was disregarded in favor of $g-r-z$ observations in order to get a more solid handle on the color of the discovered sources by covering a broader range of the optical spectrum. Assuming that a transient was detected in two of the chosen filters, even a low-significance detection or a non-detection in the third filter could be used as a metric to flag the source as a kilonova candidate based on existing models. Such information is important to prioritize spectroscopic follow-up with large telescopes.

No more DECam observations were planned on the following night(s). The total integrated probability of the LALInference skymap that we covered on the two observing nights with DECam is $67 \%$. The observations we report here are also summarized in Table 1.

\subsection{Data Analysis}

We processed and analyzed images using an imagesubtraction pipeline we developed for the discovery of GW counterparts using DECam. The pipeline, more extensively described in Goldstein et al. (2019), automatically transfers data from NOAO servers and processes them in parallel at the National Energy Research Scientific Computing Center at Lawrence Berkeley National Laboratory. Image calibration includes flat-fielding, overscan correction, and bad pixel/ column masking. The pipeline computes an astrometric solution against Gaia DR1 (Gaia Collaboration et al. 2016) sources using the Scamp package. New DECam images are aligned with reference images (or "templates") using SWarp. We use the HOTPANTS (Becker 2015) implementation of the Alard (2000) algorithm to perform image subtraction, the autoScan package to perform artifact rejection (Goldstein et al. 2015), and the PSFEx and SExtractor packages to perform point-spread function (PSF) photometry.

The pipeline obtains reference images from the DECam Legacy Survey (DECaLS) Data Release 7 and the DES Data Release 1. It calibrates photometric zero-points against the DECaLS and DES catalogs. We used the GROWTH marshal online platform (Kasliwal et al. 2019) to collect and vet transient candidates before announcing them via GCN circulars.

\section{Results \\ 4.1. Transient Candidates Discovered}

The automated pipeline described in Section 3.4 and in Goldstein et al. (2019) started yielding transient candidates $\sim 15$ minutes after the observations started at CTIO on night 1 . The LALInference skymap (The LIGO Scientific Collaboration \& the Virgo Collaboration 2019e), made available shortly after we finished the DECam observations on night 1, largely ruled out those transients as potential counterparts to S190510g. Therefore, we did not issue GCN reporting transient discoveries on night 1 and here we present only those candidates discovered on night 2 . On that night our pipeline successfully analyzed $89 \%$ of the CCDs, with the remaining $11 \%$ being unprocessed mainly due to the lack of reference images.

We vetted and prioritized candidates based on their likelihood of being extragalactic sources with host galaxies within the distance range expected for S190510g. In order to reject asteroids, our criteria for selecting candidates required two detections separated in time by $>30$ minutes. We did not include nuclear sources among the highest-priority candidates. We also excluded known transients already reported on the Transient Name Server, including SN2019bso, from the candidate list.

Using the above criteria, we narrowed down the list from 176,802 to 12 candidates and we reported them via GCN (Andreoni et al. 2019a) on 2019 May 11 02:18:53 UT, $\sim 3.5 \mathrm{hr}$ after the start of the observations. Figure 3 shows example "postage stamp" images of three of the candidates from the GCN. Table 2 summarizes relevant information and photometry of those candidates. The table includes DG19qcso 


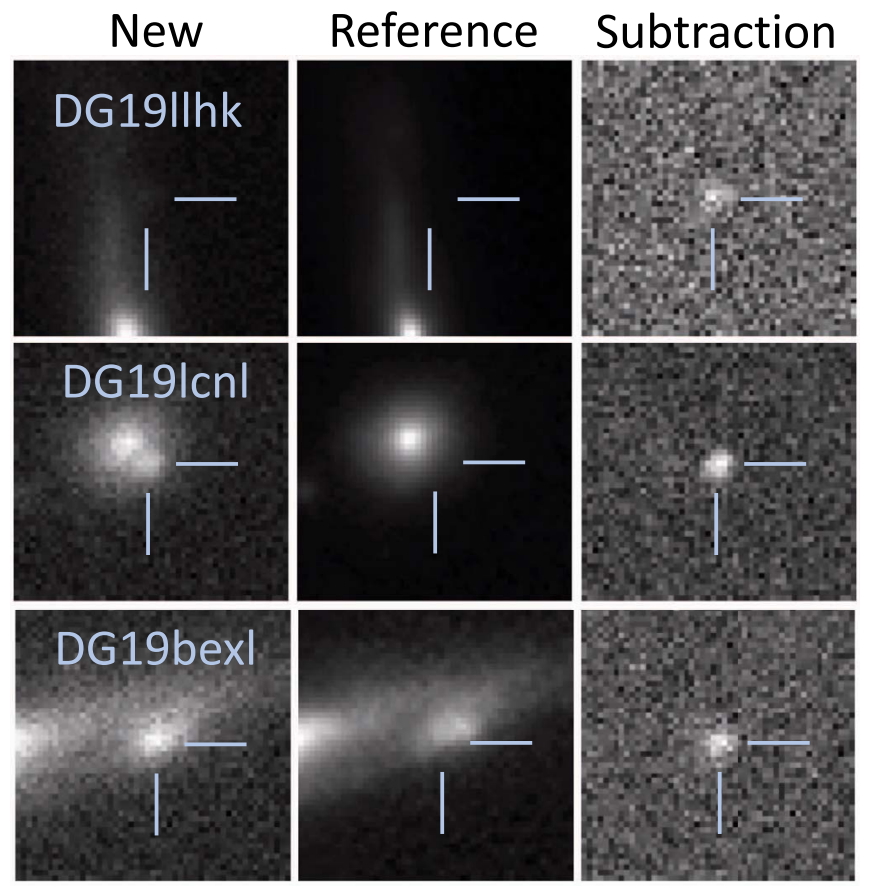

Figure 3. New image, reference image, and image subtraction of some transient candidates discovered with our image-subtraction pipeline (Goldstein et al. 2019). The side of each squared "postage stamp" measures 13 ". 2 . The complete poll of candidates selected by our program during the follow-up of S190510g is presented in Section 4.1 and in Tables 2-3.

(where the prefix "DG" indicates the detection of the candidate by the "DECam-GROWTH" project), a transient candidate that met the above criteria, but that was reported in a separate GCN circular (Kool et al. 2019). In Table 3 we report other candidates that appear to be nuclear, hostless, or with faint host galaxies likely placed at large distances for completeness.

Two of the reported transients seemed to be of particular interest. The first candidate, DG191lhk, appeared to be associated with a host galaxy located at redshift $z=0.07158$ according to the $6 \mathrm{dF}$ galaxy survey (Jones et al. 2009). The redshift was consistent with the LVC distance estimate. At that distance, the absolute magnitude of DG191lhk was -16.4 , consistent with GW170817 at +1 day. The second candidate of interest was DG19lcnl, whose color from preliminary photometry $\quad\left(m_{g}=20.27 \pm 0.04, \quad m_{r}=19.45 \pm 0.02\right.$, $\left.m_{z}=20.18 \pm 0.06\right)$ appeared consistent with GW170817 at the same phase. The lack of a coincident source in the reference image and the proximity of the transient to a galaxy (Figure 3) suggested the nature of DG19lcnl to be extragalactic, although a redshift of the putative host was not present in survey catalogs.

Photometric follow-up with the Korea Microlensing Telescope Network (KMTNet, Kim et al. 2016) between 2019 May 10 16:48:24 UT and before 2019 May 11 23:42:11 UT indicated no significant fading $\left(\Delta R>0.5\right.$ mag day $\left.^{-1}\right)$ for any of the 12 transients reported by Andreoni et al. (2019a) (including DG191lhk and DG19lcnl) or with respect to our preliminary $r$-band DECam photometry (Im et al. 2019a, 2019b, 2019c). A decay of $\Delta r \gtrsim 0.5 \mathrm{mag} \mathrm{day}^{-1}$ is expected from kilonovae at this phase, thus the KMTNet photometry provided evidence against any of the 12 transients in the GCN being the counterpart to S190510g.

Gomez et al. (2019) used the IMACS Spectrograph on the $6.5 \mathrm{~m}$ Magellan-Baade telescope to spectroscopically classify the candidate DG19fqqk as a SN II at redshift $z=0.06$, confirming that the source was unrelated to the GW event. Observations performed after the GW trigger time, with the X-ray Telescope (XRT) on board the Neil Gehrels Swift Observatory, revealed no X-ray source at the location of DG19fqqk with a $3 \sigma$ upper limit of $6.1 \times 10^{-2} \mathrm{ct} \mathrm{s}^{-1}$, corresponding to a $0.3-10 \mathrm{keV}$ flux of $2.6 \times 10^{-12} \mathrm{erg} \mathrm{cm}^{-2} \mathrm{~s}^{-1}$ (Evans et al. 2019). XRT upper limits are also available for the candidate DG191lhk, for which no X-ray flux was detected down to $6.4 \times 10^{-2}$ ct s$^{-1}(3 \sigma)$, corresponding to $2.8 \times 10^{-12} \mathrm{erg} \mathrm{cm}^{-2} \mathrm{~s}^{-1}$ (Evans et al. 2019).

The DES Collaboration reported 11 transients from an independent analysis the public DECam data (Annis 2019; Soares-Santos 2019). Six of those candidates confirmed detections already reported in Andreoni et al. (2019a) and one corresponded to the known supernova SN2019bso. The four remaining candidates were not reported by our team. The desgw-190510a and desgw-190510f candidates were detected only once by our pipeline (thus not passing our selection criteria), while desgw-190510b and desgw-190510g were not detected in any band. In particular, desgw-190510a was detected by our pipeline in the $r$ band $(r=20.980 \pm 0.065)$, but it was not detected in the $g$ band (faint, $g>21.8$ ) and the $z$ band (rejected by our automatic cuts on the candidate shape). The candidate desgw-190510b had no template coverage in DES DR1. The candidate desgw-190510g was not detected in the $g$ band (rejected) and $z$ band $(z>21.4)$, with no DES DR1 coverage available in the $r$ band. Finally, desgw-190510f was detected by our pipeline in the $r$ band $(r=21.296 \pm 0.051)$, but it was not detected in the $g$ band (rejected) and $z$ band (faint, $z>20.8$ ).

\subsection{Observing Efficiency}

We define the efficiency $\epsilon$ of our observations as the ratio of the time spent on sky (accounting for the minimum overhead per exposure, mostly dictated by the CCD readout) over the total duration of the observations

$$
\epsilon=\frac{n_{\exp } \times(\text { exptime }+ \text { overhead })}{t_{\text {tot }}}
$$

where $n_{\text {exp }}$ represents the number of exposures, "overhead" is the minimum overhead possible per exposure, and $t_{\text {tot }}$ is the duration of the observations. Such a definition of efficiency is also valid outside the GW follow-up context.

Assuming $20 \mathrm{~s}$ of CCD readout to be the dominant overhead, we obtained an efficiency $\epsilon=0.70$ on night 1 and $\epsilon=0.80$ on night 2 . More realistically, the overhead time for each exposure amounts to $\sim 30 \mathrm{~s}$ even without any slewing (see for example Andreoni et al. 2019c), in which case the efficiency increases to $\epsilon=0.84$ on night 1 and $\epsilon=0.94$ on night 2 . The lower efficiency of night 1 is almost entirely due to two outliers in the distribution of slewing times. A CTIO computer failure caused a loss of 13.8 minutes starting at 2019 May 10 08:08 UT. A $100.2 \mathrm{~s}$ slew between the end of the $g$-band observing block and the beginning of the $z$-band observing block constitutes the second outlier. Removing those outliers, the efficiency between night 1 and night 2 is comparable within $<2 \%$, with the difference due to larger slews dictated by the larger area to cover (in other words, by the less precise localization skymap).

The observing efficiency using the greedy-slew algorithm was superior to the greedy algorithm for DECam. During the 
Table 2

Transient Candidates Discovered with Our Automated Pipeline (Goldstein et al. 2019) in the Public DECam Data That We Acquired

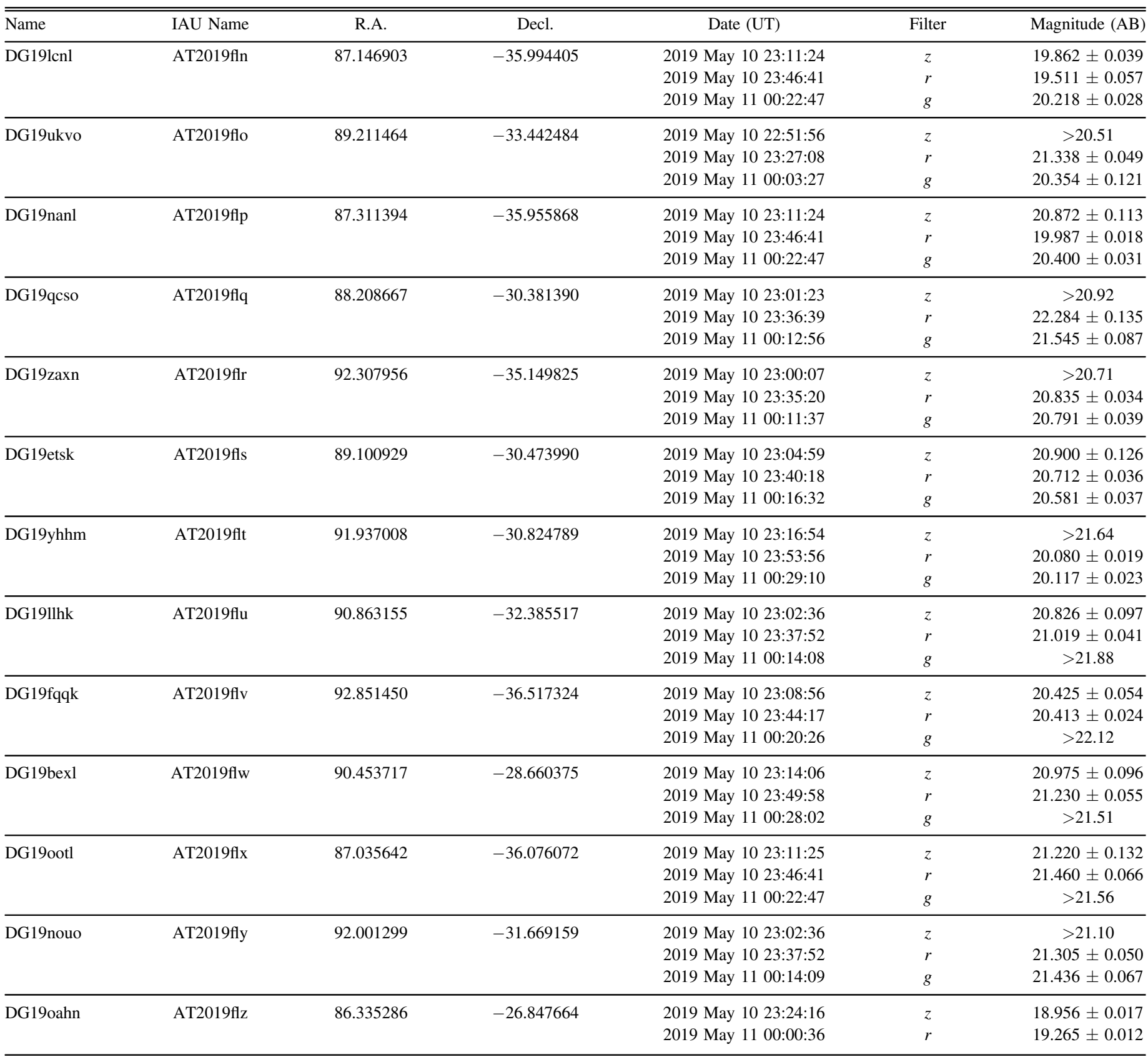

Note. The prefix "DG" of the candidate names indicates their detection within the "DECam-GROWTH" project. The observing date corresponds to the start of the exposures. The photometric measurements are not corrected for galactic extinction. These candidates (other than DG19qcso, Kool et al. 2019) were reported $\sim 3$ hr after the beginning of the observations Andreoni et al. (2019a) and have higher priority. All the candidates (including DG19qcso) are likely associated with prominent host galaxies.

DECam follow-up of the GW event S190426c (Goldstein et al. 2019) the overall efficiency of our observations was $\epsilon=0.52$ and $\epsilon=0.60$ assuming $20 \mathrm{~s}$ and $\sim 30 \mathrm{~s}$ overhead per exposure respectively. The greedy-slew algorithm optimized for DECam, along with a better (more conservative) overhead time estimation, led to a significant improvement during the follow-up of S190510g. Those new techniques were developed in $<2$ weeks by the GROWTH ToO marshal team, after S190426c and before S190510g happened.

\section{Discussion}

The follow-up of S190510g highlighted the trade-off between depth, area to cover, and color information that must be faced during GW follow-up of BNS mergers during O3. The event had a very large localization area on night 1 (3462 deg for the $90 \%$ probability) and its initial distance was at least three times the distance to GW170817. At such large distances, the expected kilonova emission may be too faint to be detected with $1 \mathrm{~m}$ class telescopes and galaxy-targeted follow-up becomes less efficient due to poorer galaxy catalog 
Table 3

Same as Table 2, but Presenting Lower-priority Candidates That Did Not Meet the Criteria That We Described in Section 4.1

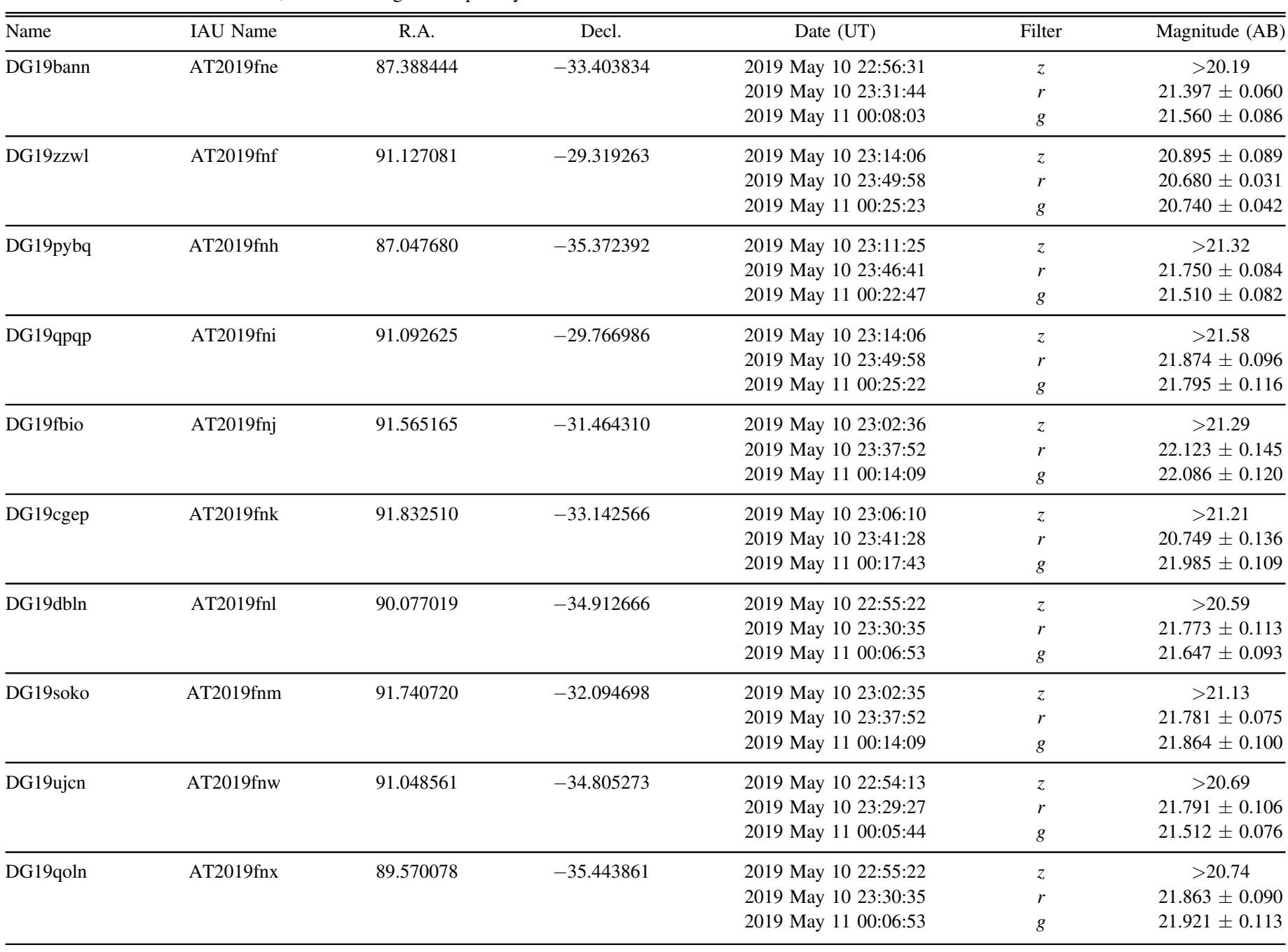

Note. The low-priority candidates are reported here for completeness.

completeness and larger numbers of galaxies enclosed in the highest-probability volume.

Covering large sky areas, as in the case of S190510g (and S190426c before that), is made possible by optimized algorithms (see Section 3.1) that push the observations close to the physical limits of the telescope mount. Our new greedyslew algorithm was able to achieve a total of $\epsilon=94 \%$ efficiency and up to $\epsilon=99 \%$ excluding filter changes on the second observing night. If the telescope, the dome, or the observing software experiences issues that causes the observations to stop temporarily (as it happened on night 1 of the observations presented here) the schedule should be recomputed in real time.

The follow-up of S190510g also highlighted the relevance of DECam observations when the highest-probability localization region is located at decl. $<-30^{\circ}$, too far south for many northern hemisphere survey facilities (including, for example, ZTF, ATLAS, and Pan-STARRS) to observe. High-probability skymap regions were observable from CTIO on both night 1 and night 2, even after the skymap change.

In Figure 4 we compare the limiting magnitudes of night 2 with a sample of kilonova models (Kasen et al. 2017; Bulla et al. 2019; Bulla 2019). The $3 \sigma$ limiting magnitudes of $g<21.7, r<22.3$, and $z<21.2$ were calculated by estimating the magnitude of a source with a count rate per pixel three times higher than the sky background. With $40 \mathrm{~s}$ exposure time in $g-r-z$ bands, we could have detected a GW170817-like kilonova (Figure 4, upper panel, based on Kasen et al. 2017) in at least two filters out to $\sim 227 \mathrm{Mpc}$, the center of the distance distribution inferred by the LALInference skymap $(227 \pm 92 \mathrm{Mpc}$, The LIGO Scientific Collaboration \& the Virgo Collaboration 2019e).

Other models (Figure 4, lower panels, based on Bulla et al. 2019; Bulla 2019) allow us to explore the detectability of a GW170817-like kilonova at different viewing angles. The comparison of the models with the DECam detection limits suggests good detection chances in two or three filters out to $\sim 230 \mathrm{Mpc}$ under polar viewing angles (i.e., when the kilonova is viewed face-on), with the identification of a counterpart becoming more difficult at equatorial viewing angles. We stress that the non-detection in one band can add important information to help reject contaminants (such as supernovae) and lead to rapid identification of the most promising kilonova candidates. We note that the observations of GW170817 provided well-sampled, multi-wavelength light curves that we could use to better plan our follow-up campaigns and to assess 

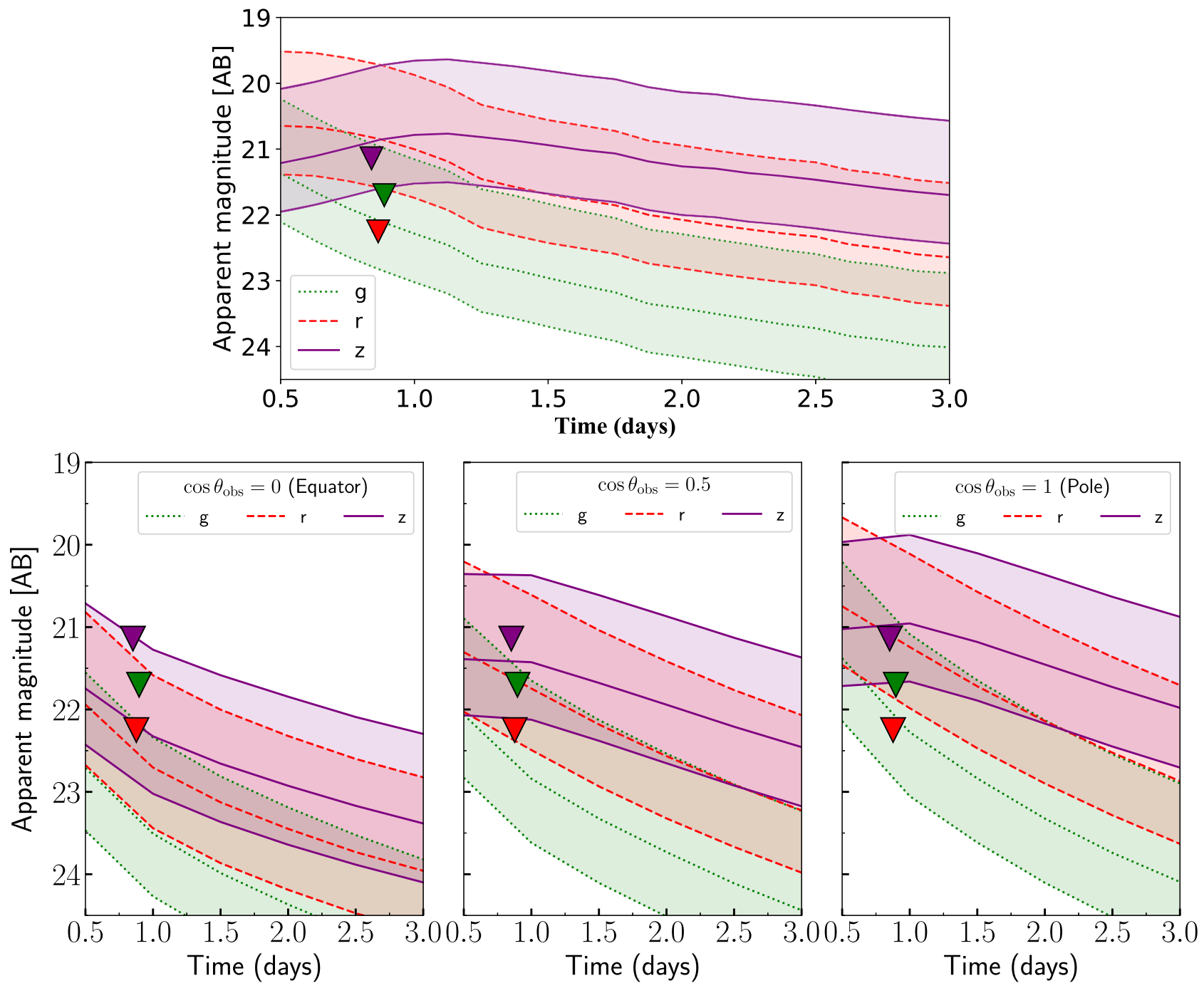

Figure 4. Kilonova models developed by Kasen et al. (2017) (upper panel) and Bulla et al. (2019), and Bulla (2019) (lower panels). All those models account for two kilonova components, a "blue" lanthanide-poor and a "red" lanthanide-rich component. Triangles indicate the median-limiting magnitudes of our observations. In the upper panel, the GW170817-like kilonova is placed at the distance range expected for S190510g. In the lower panel, the models by Bulla et al. (2019) and Bulla (2019) show what the GW170817 kilonova emission would look like at three different viewing angles (from edge-on, $\cos \theta_{\mathrm{obs}}=0$, to face-on, $\cos \theta_{\mathrm{obs}}=1$ ). The extension of the lanthanide-rich component around the equatorial/merger plane is parameterized by an half-opening angle set to $\phi=30^{\circ}$, which best fits the observed light curve of GW170817 (Bulla 2019). The plots presented in this figure show that we would have likely been able to detect a kilonova with the same properties of GW170817 in at least two bands at the central distance of the distribution inferred from the GW data analysis under favorable viewing angles.

the results of our kilonova searches in real time. However, the kilonova population is likely diverse, as recent (postGW170817) studies of short gamma-ray burst optical afterglows suggest (Fong et al. 2017; Gompertz et al. 2018; Troja et al. 2018; Rossi et al. 2019). The discovery of a larger population of kilonovae will allow us to get a more solid handle on the distribution of their physical and observational properties. Key to the detection of more GW optical counterparts is the combination of deep imaging and wide-area coverage, which our DECam observations aim to maximize. The upcoming Large Synoptic Survey Telescope is expected to be more sensitive than DECam, and thanks to its $\sim 10 \mathrm{deg}^{2}$ field of view, may allow us to probe the fainter end of the kilonova luminosity distribution even for those mergers localized over an area of hundreds of $\mathrm{deg}^{2}$.

Optical counterpart candidates discovered with our automatic pipeline (Section 3.4) were carefully vetted and made public $\sim 3 \mathrm{hr}$ after the beginning of the observations (Section 4). This enabled multi-wavelength photometric and spectroscopic follow-up to be promptly performed on candidates that we and other groups prioritized. Candidates could be made available in batches every $\sim 2 \mathrm{hr}$ during future follow-ups.

\section{Conclusion}

In this paper we presented our DECam follow-up of S190510g, a possible BNS merger discovered by LVC. The observations were planned using the GROWTH ToO marshal scheduling platform (Coughlin et al. 2019; Kasliwal et al. 2019) using algorithms that we have optimized specifically for DECam, but that could be applied to other facilities. On the second night of our DECam follow-up of S190510g we covered $65 \%$ of the localization probability. We reached a total observing efficiency of $\epsilon=0.94$, which was a marked improvement over our first DECam GW follow-up of O3 ( $\epsilon=0.60$, Goldstein et al. 2019).

Our observations were deeper than planned in our baseline strategy to account for the distance to the event larger than 
expected, possibly as large as $\sim 8$ times the distance to GW170817. We estimate that our observations can lead to the detection of GW170817-like kilonovae in at least two bands at $\sim 230 \mathrm{Mpc}$ over an area of $75 \mathrm{deg}^{2}$ in the $1.66 \mathrm{hr}$ of observing time that was available, albeit under favorable viewing angles.

Additional information such as constraints on the viewing angle from early multi-messenger analysis could help with choosing the most appropriate combination of filters, cadence, and exposure times for future electromagnetic follow-ups (see, for example, Chen et al. 2018). Coarsely localized GW events at (or beyond) the angle-averaged horizon of the Advanced LIGO and Virgo detectors may be common during O3, thus optimally scheduled observations with DECam can be key in upcoming follow-up of new GW triggers.

On behalf of the entire GROWTH team, I.A. and D.A.G. gratefully acknowledge Steve Heathcote, Kathy Vivas, Tim Abbott, and the staff at CTIO and NOAO for facilitating these target-of-opportunity observations. I.A. and M.M.K. thank Myungshin Im and the KMTNet team for coordinating followup observations of DECam-GROWTH candidates. The authors thank the anonymous referee for a useful review of the manuscript.

This work was supported by the GROWTH (Global Relay of Observatories Watching Transients Happen) project funded by the National Science Foundation under PIRE grant No. 1545949. GROWTH is a collaborative project among California Institute of Technology (USA), University of Maryland College Park (USA), University of Wisconsin Milwaukee (USA), Texas Tech University (USA), San Diego State University (USA), University of Washington (USA), Los Alamos National Laboratory (USA), Tokyo Institute of Technology (Japan), National Central University (Taiwan), Indian Institute of Astrophysics (India), Indian Institute of Technology Bombay (India), Weizmann Institute of Science (Israel), The Oskar Klein Centre at Stockholm University (Sweden), Humboldt University (Germany), Liverpool John Moores University (UK), and University of Sydney (Australia).

D.A.G. acknowledges support from Hubble Fellowship grant HST-HF2-51408.001-A. S.A. acknowledges support from the PMA Division Medberry Fellowship at the California Institute of Technology. Support for Program number HST-HF251408.001-A is provided by NASA through a grant from the Space Telescope Science Institute, which is operated by the Association of Universities for Research in Astronomy, Incorporated, under NASA contract NAS5-26555. M.W.C. is supported by the David and Ellen Lee Postdoctoral Fellowship at the California Institute of Technology. M.B. and E.K. acknowledge support from the G.R.E.A.T. research environment funded by the Swedish National Science Foundation. P. N. acknowledges support from the DOE through DE-FOA0001088, Analytical Modeling for Extreme-Scale Computing Environments. J.S.B., J.M.-P., and K.Z. are partially supported by a Gordon and Betty Moore Foundation Data-Driven Discovery grant. Support for J.B. was provided in part by the National Aeronautics and Space Administration(NASA) through the Einstein Fellowship Program, grant No. PF7180162. P.G. is supported by NASA Earth and Space Science Fellowship (ASTRO18F-0085). A.A.M. acknowledges support from the following grants: NSF AST-1749235, NSF-1640818 and NASA 16-ADAP16-0232. V.Z.G. acknowledges support from the University of Washington College of Arts and
Sciences, Department of Astronomy, and the DIRAC Institute. University of Washington's DIRAC Institute is supported through generous gifts from the Charles and Lisa Simonyi Fund for Arts and Sciences, and the Washington Research Foundation. A.K.H.K. acknowledges support from the Ministry of Science and Technology of the Republic of China (Taiwan) under grants 106-2628-M-007-005 and 107-2628-M-007-003.

This project used data obtained with the Dark Energy Camera (DECam), which was constructed by the Dark Energy Survey (DES) collaborating institutions: Argonne National Lab, University of California Santa Cruz, University of Cambridge, Centro de Investigaciones Energeticas, Medioambientales y Tecnologicas-Madrid, University of Chicago, University College London, DES-Brazil consortium, University of Edinburgh, ETH-Zurich, University of Illinois at Urbana-Champaign, Institut de Ciencies de l'Espai, Institut de Fisica d'Altes Energies, Lawrence Berkeley National Lab, Ludwig-Maximilians Universitat, University of Michigan, National Optical Astronomy Observatory, University of Nottingham, Ohio State University, University of Pennsylvania, University of Portsmouth, SLAC National Lab, Stanford University, University of Sussex, and Texas A\&M University. Funding for DES, including DECam, has been provided by the U.S. Department of Energy, National Science Foundation, Ministry of Education and Science (Spain), Science and Technology Facilities Council (UK), Higher Education Funding Council (England), National Center for Supercomputing Applications, Kavli Institute for Cosmological Physics, Financiadora de Estudos e Projetos, Fundação Carlos Chagas Filho de Amparo a Pesquisa, Conselho Nacional de Desenvolvimento Científico e Tecnológico and the Ministério da Ciência e Tecnologia (Brazil), the German Research Foundation-sponsored cluster of excellence "Origin and Structure of the Universe" and the DES collaborating institutions.

This work has made use of data from the European Space Agency (ESA) mission Gaia (Gaia Collaboration et al. 2016, 2018) (https://www.cosmos.esa.int/gaia), processed by the Gaia Data Processing and Analysis Consortium (DPAC, https://www.cosmos.esa.int/web/gaia/dpac/ consortium). Funding for the DPAC has been provided by national institutions, in particular the institutions participating in the Gaia Multilateral Agreement.

\section{ORCID iDs}

Igor Andreoni (1D https://orcid.org/0000-0002-8977-1498 Daniel A. Goldstein (iD https://orcid.org/0000-0003-3461-8661 Michael W. Coughlin (1) https://orcid.org/0000-00028262-2924

Leo P. Singer (iD https://orcid.org/0000-0001-9898-5597 Tomás Ahumada (iD https://orcid.org/0000-0002-2184-6430 Michael Medford (i) https://orcid.org/0000-0002-7226-0659 Erik C. Kool (i) https://orcid.org/0000-0002-7252-3877 Sara Webb (ib https://orcid.org/0000-0003-2601-1472 Mattia Bulla (ib https://orcid.org/0000-0002-8255-5127 Joshua S. Bloom (iD https://orcid.org/0000-0002-7777-216X Mansi M. Kasliwal (iD https://orcid.org/0000-0002-5619-4938 Peter E. Nugent (1) https://orcid.org/0000-0002-3389-0586 Jennifer Barnes (i) https://orcid.org/0000-0003-3340-4784 David O. Cook (iD https://orcid.org/0000-0002-6877-7655 Jeff Cooke (iD https://orcid.org/0000-0001-5703-2108 Dmitry A. Duev (10 https://orcid.org/0000-0001-5060-8733 Pradip Gatkine (iD https://orcid.org/0000-0002-1955-2230 
V. Zach Golkhou (1) https://orcid.org/0000-0001-8205-2506

Albert K. H. Kong (1) https://orcid.org/0000-0002-5105-344X

Ashish Mahabal (10 https://orcid.org/0000-0003-2242-0244

Keming Zhang

(张可名) (1) https://orcid.org/0000-0002-9870-5695

\section{References}

Abbott, B., Abbott, R., Abbott, T. D., et al. 2017a, Natur, 551, 85 Abbott, B., Abbott, R., Abbott, T. D., et al. 2017b, PhRvL, 119, 161101 Abbott, B., Abbott, R., Abbott, T. D., et al. 2017c, ApJL, 848, L12 Abbott, B., Abbott, R., Abbott, T. D., et al. 2018, LRR, 21, 3 Alard, C. 2000, A\&AS, 144, 363

Andreoni, I., Cooke, J., Webb, S., et al. 2019c, arXiv:1903.11083 Andreoni, I., Goldstein, D. A., Anand, S., et al. 2019a, GCN, 24467, 1 Andreoni, I., Goldstein, D. A., Coughlin, M. W., et al. 2019b, GCN, 24443, 1 Annis, J. 2019, GCN, 24474, 1

Annis, J., Soares-Santos, M., Berger, E., et al. 2016, ApJL, 823, L34 Arcavi, I., Hosseinzadeh, G., Howell, D. A., et al. 2017, Natur, 551, 64 Barnes, J., Kasen, D., Wu, M.-R., \& Martínez-Pinedo, G. 2016, ApJ, 829, 110 Becker, A. 2015, HOTPANTS: High Order Transform of PSF ANd Template Subtraction, Astrophysics Source Code Library, ascl:1504.004 Bellm, E. C., Kulkarni, S. R., Graham, M. J., et al. 2019, PASP, 131, 018002 Bulla, M. 2019, arXiv: 1906.04205

Bulla, M., Covino, S., Kyutoku, K., et al. 2019, NatAs, 3, 99

Cantiello, M., Jensen, J. B., Blakeslee, J. P., et al. 2018, ApJL, 854, L31

Chen, H.-Y., Vitale, S., \& Narayan, R. 2018, arXiv:1807.05226

Chornock, R., Berger, E., Kasen, D., et al. 2017, ApJL, 848, L19

Cook, D. O., Kasliwal, M. M., Van Sistine, A., et al. 2019, ApJ, 880, 7

Coughlin, M. W., Ahumada, T., Cenko, S. B., et al. 2019, PASP, 131, 048001

Coughlin, M. W., Tao, D., Chan, M. L., et al. 2018, MNRAS, 478, 692

Coulter, D. A., Foley, R. J., Kilpatrick, C. D., et al. 2017, Sci, 358, 1556

Cowperthwaite, P. S., Berger, E., Soares-Santos, M., et al. 2016, ApJL, 826, L29

Cowperthwaite, P. S., Berger, E., Villar, V. A., et al. 2017, ApJL, 848, L17

Doctor, Z., Kessler, R., Herner, K., et al. 2019, ApJL, 873, L24

Evans, P. A., Kennea, J. A., Tohuvavohu, A., et al. 2019, GCN, 24541, 1

Flaugher, B., Diehl, H. T., Honscheid, K., et al. 2015, AJ, 150, 150

Fong, W., Berger, E., Blanchard, P. K., et al. 2017, ApJL, 848, L23

Gaia Collaboration, Brown, A. G. A., Vallenari, A., et al. 2018, A\&A, 616, A1

Gaia Collaboration, Prusti, T., de Bruijne, J. H. J., et al. 2016, A\&A, 595, A1

Goldstein, D. A., Andreoni, I., Nugent, P. E., et al. 2019, ApJL, in press (arXiv:1905.06980)

Goldstein, D. A., D'Andrea, C. B., Fischer, J. A., et al. 2015, AJ, 150, 82

Golkhou, V. Z., Butler, N. R., Strausbaugh, R., et al. 2018, ApJ, 857, 81
Gomez, S., Cowperthwaite, P. S., Hosseinzadeh, G., et al. 2019, GCN, 24511, 1

Gompertz, B. P., Levan, A. J., Tanvir, N. R., et al. 2018, ApJ, 860, 62

Hjorth, J., Levan, A. J., Tanvir, N. R., et al. 2017, ApJL, 848, L31

Im, M., Kim, J., Lee, C.-U., et al. 2019a, GCN, 24529, 1

Im, M., Kim, J., Lee, C.-U., et al. 2019b, GCN, 24466, 1

Im, M., Kim, J., Lee, C.-U., et al. 2019c, GCN, 24493, 1

Im, M., Yoon, Y., Lee, S.-K. J., et al. 2017, ApJL, 849, L16

Jones, D. H., Read, M. A., Saunders, W., et al. 2009, MNRAS, 399, 683

Kasen, D., Metzger, B., Barnes, J., Quataert, E., \& Ramirez-Ruiz, E. 2017, Natur, 551, 80

Kasliwal, M. M., Cannella, C., Bagdasaryan, A., et al. 2019, PASP, 131, 038003

Kasliwal, M. M., Nakar, E., Singer, L. P., et al. 2017, Sci, 358, 1559

Kilpatrick, C. D., Foley, R. J., Kasen, D., et al. 2017, Sci, 358, 1583

Kim, S.-L., Lee, C.-U., Park, B.-G., et al. 2016, JKAS, 49, 37

Kool, E., Goldstein, D. A., \& Andreoni, I. 2019, GCN, 24540, 1

Levan, A. J., Lyman, J. D., Tanvir, N. R., et al. 2017, ApJL, 848, L28

Lipunov, V., Gorbovskoy, E., Kornilov, V. G., et al. 2017, ApJL, 850, L1

Messick, C., Blackburn, K., Brady, P., et al. 2017, PhRvD, 95, 042001

Pan, Y.-C., Kilpatrick, C. D., Simon, J. D., et al. 2017, ApJL, 848, L30

Pian, E., D'Avanzo, P., Benetti, S., et al. 2017, Natur, 551, 67

Rana, J., Anand, S., \& Bose, S. 2019, ApJ, 876, 104

Rana, J., Singhal, A., Gadre, B., Bhalerao, V., \& Bose, S. 2017, ApJ, 838, 108

Rossi, A., Stratta, G., Maiorano, E., et al. 2019, arXiv:1901.05792

Shappee, B. J., Simon, J. D., Drout, M. R., et al. 2017, Sci, 358, 1574

Singer, L. P., \& Price, L. R. 2016, PhRvD, 93, 024013

Smartt, S. J., Chen, T. W., Jerkstrand, A., et al. 2017, Natur, 551, 75

Soares-Santos, M. 2019, GCN, 24480, 1

Soares-Santos, M., Holz, D. E., Annis, J., et al. 2017, ApJL, 848, L16

Soares-Santos, M., Kessler, R., Berger, E., et al. 2016, ApJL, 823, L33

Tanvir, N. R., Levan, A. J., González-Fernández, C., et al. 2017, ApJL, 848, L27

The LIGO Scientific Collaboration \& the Virgo Collaboration 2019a, GCN, 24168, 1

The LIGO Scientific Collaboration \& the Virgo Collaboration 2019b, GCN, 24237, 1

The LIGO Scientific Collaboration \& the Virgo Collaboration 2019c, GCN, 24442, 1

The LIGO Scientific Collaboration \& the Virgo Collaboration 2019d, GCN, 24489, 1

The LIGO Scientific Collaboration \& the Virgo Collaboration 2019e, GCN, 24448, 1

Troja, E., Ryan, G., Piro, L., et al. 2018, NatCo, 9, 4089

Valenti, S., Sand, D. J., Yang, S., et al. 2017, ApJL, 848, L24

Veitch, J., Raymond, V., Farr, B., et al. 2015, PhRvD, 91, 024013042003 